\title{
Heme oxygenase-1 protects $\mathrm{H}_{2} \mathrm{O}_{2}$-insulted glomerular mesangial cells from excessive autophagy
}

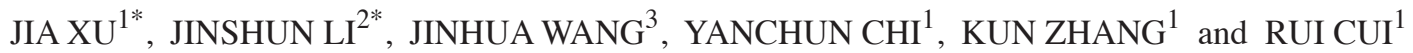 \\ ${ }^{1}$ Department of Nephrology, The Fourth Affiliated Hospital, Harbin Medical University, Harbin, Heilongjiang 150001; \\ ${ }^{2}$ Department of Cardiology, The Nangang Branch Hospital of Heilongjiang Provincial Hospital, \\ Nangang, Heilongjiang 150000; ${ }^{3}$ Department of Pharmacy Intravenous Admixture Service, \\ The First Affiliated Hospital of Harbin Medical University, Harbin, Heilongjiang 572000, P.R. China
}

Received May 29, 2015; Accepted April 8, 2016

DOI: $10.3892 / \mathrm{mmr} .2016 .5177$

\begin{abstract}
Increasing evidence has demonstrated that the activation of heme oxygenase (HO)-1 reduces autophagy stimulated by oxidative stress injury, in which the supraphysiological production of reactive oxygen species (ROS) is detected. However, the potential mechanism underlying this effect remains unclear. The present study aimed to investigate the function of HO-1 activation in the regulation of autophagy in glomerular mesangial cells subjected to $\mathrm{H}_{2} \mathrm{O}_{2}$-induced oxidative stress injury. The results demonstrated that the HO-1 agonist, hemin, reduces the LC3 protein level, which was enhanced by $\mathrm{H}_{2} \mathrm{O}_{2}$ treatment. Furthermore, hemin-activated HO-1 may function as a regulator of oxidative stress-induced autophagy in a dose-dependent manner. Pharmacological activation of c-Jun N-terminal kinase (JNK) inhibited the effect of hemin, indicating that the JNK signaling pathway is associated with the mechanism of HO-1 in impeding excessive autophagy. In addition to successfully alleviating $\mathrm{H}_{2} \mathrm{O}_{2}$-induced oxidative stress and cellular apoptosis, hemin-activated HO-1 may provide cytoprotection against rapamycin, a specific autophagy agonist. The present result suggested the inhibitory action of HO-1 in the avoidance of a potentially enhanced linkage between autophagy and apoptosis, particularly in the setting of excessive ROS. Therefore, enhancing the intracellular activity of HO-1 may assist the crosstalk between oxidative stress, autophagy and apoptosis, and represent a novel therapeutic strategy for renal ischemic disease.
\end{abstract}

Correspondence to: Dr Rui Cui, Department of Nephrology, The Fourth Affiliated Hospital, Harbin Medical University, 37 Yiyuan Street, Harbin, Heilongjiang 150001, P.R. China

E-mail: 55570816@qq.com

${ }^{*}$ Contributed equally

Key words: heme oxygenase-1, oxidative stress, autophagy, apoptosis, glomerular mesangial cell

\section{Introduction}

Previous investigation has demonstrated that heme oxygenase (HO), particularly the stress-responsive isoenzyme HO-1, exhibits vital regulatory functions in renal processes under pathophysiological conditions (1). Due to its beneficial effects, including oxidative stress relief and the inhibition of cell apoptosis, HO-1 has previously been proposed as a potential therapeutic target for ischemia/reperfusion or hypertension-induced glomerular injuries $(2,3)$, and diabetic retinopathy (4). HO-1 has also been considered a potential biomarker of acute kidney injury (5). Multiple mechanisms may be involved in the protective effects of activated HO-1 (6). In a previous study, the effect of HO-1 in autophagy, an apoptosis-associated biological process, was also investigated, and an implication of the comprehensive understanding of the protective mechanisms of HO-1 was provided (7).

Autophagy has been widely studied in the last decade (8). The mechanism of autophagy has been elucidated based on pathophysiology to understand human biology and disease (9-11). Autophagy must be reconsidered not only as a self-degrading strategy for cell survival, but also as a type of dynamic and systematic re-organization of cellular materials and energy (12). As such, an appropriate intervention strategy effecting autophagy may direct cellular materials and energy flow to intended processes in order to result in the alleviation of diseases. Therefore, targeting autophagy may be a non-limiting therapeutic strategy for human disease prevention and treatment. However, despite evidence of the cytoprotective effects provided by enhanced autophagy (13), excessive autophagic flux induces apoptotic damage to cells (14). This previous finding demonstrates that prudent consideration is required prior to proposing autophagy-driven treatment strategies.

In renal pathology (15), HO-1 inhibits autophagy in acute kidney injury and functions as an established therapeutic target for renal diseases (3). However, the regulatory mechanism through which HO-1 inhibits autophagy remains unclear (7). It is currently recognized that the c-Jun N-terminal kinase (JNK) signaling pathway is important for modulating the crosstalk between oxidative stress and autophagy. This finding explains the co-existence of JNK activation and enlarged autophagic flux observed in the intracellular setting during 
reactive oxygen species (ROS) overload (16). In accordance with this observation, a previous study demonstrated that the inhibition of JNK, but not p38, prevents unsaturated aldehyde-induced HO-1 expression and microtubule associated protein 1 light chain 3 (LC3)-II-mediated autophagy (17). Thus, the LC3 II/LC3 I expression ratio was used as a marker of autophagy in the present study (9). In addition, p38 inhibition affects nuclear translocation of the HO-1 transcription factor, nuclear factor erythroid 2 like 2, by reducing the intracellular accumulation of p62 (18). Taken together, these previous findings indicate that the JNK signaling pathway is crucial in ROS-induced HO-1 expression. However, it remains unclear whether HO-1-mediated autophagy inhibition is also associated with JNK signaling in cells insulted by excessive ROS, including $\mathrm{H}_{2} \mathrm{O}_{2}$-induced glomerular mesangial cells. Furthermore, based on the demonstrated effect of HO-1 on the inhibition of ROS-induced autophagy (3), it is also important to identify the function of HO-1 in this setting. Intrinsically, the biological importance of HO-1 remains unclear, however, it may provide oxidative stress relief and simultaneously inhibit autophagy. The present study aimed to determine the function of HO-1 during complex biological processes, including the oxidative stress response, autophagy and apoptosis, and whether application of HO-1 for the prevention and treatment of kidney disease is plausable.

\section{Materials and methods}

Reagents, cell culture and $\mathrm{H}_{2} \mathrm{O}_{2}$ treatment. Hemin, zinc protoporphyrin (ZnPP) and rapamycin were purchased from Sigma-Aldrich (St. Louis, MO, USA). The JNK agonist, anisomycin, and the rabbit anti-LC3 antibodies (cat. no. 3868) were obtained from Merck Millipore (Darmstadt, Germany) and Cell Signaling Technology, Inc. (Danvers, MA, USA), respectively. An established stable human glomerular mesangial cell line (donated by Dr JD Sraer, Hopital Tenon, Paris, France) was cultured as described previously (19). Briefly, these cells were cultured in RPMI-1640 medium (Gibco; Thermo Fisher Scientific, Inc., Waltham, MA, USA) supplemented with Gibco $10 \%$ fetal bovine serum (FBS; Thermo Fisher Scientific, Inc.). Cells between passage 3 and 15 were used and then subcultured in serum-deprived medium (1\% FBS) for $12 \mathrm{~h}$ prior to further experiments. The cells were treated with $125 \mu \mathrm{M} \mathrm{H}_{2} \mathrm{O}_{2}$ for $24 \mathrm{~h}$.

Cell viability assay. The cells were seeded into 96-well culture plates at a density of $2.5 \times 10^{4}$ cells/well, and incubated at $37^{\circ} \mathrm{C}$ with $5 \% \mathrm{CO}_{2}$. Following treatment of the cells with the $\mathrm{H}_{2} \mathrm{O}_{2}$ or/anddrugtreatments, a3-(4,5-dimethyl-2-thiazolyl)-2,5-diphenyl-2-H-tetrazolium bromide (MTT) assay (Sigma-Aldrich) was performed. Briefly, $20 \mu \mathrm{l}$ MTT solution was added to each well and the cells were incubated for $4 \mathrm{~h}$. Formazan crystals were subsequently dissolved in $150 \mu \mathrm{l}$ dimethyl sulfoxide. The optical density of the wells was measured at $490 \mathrm{~nm}$ (Bio-TEK Epoch; BioTek Instruments, Inc., Winooski, VT, USA).

Caspase-3 activity assay. To assess the activity of caspase-3, the mesangial cells were scraped from the plates in ice-cold phosphate-buffered saline (PBS). The cells were subsequently lysed in $160 \mu 1$ ice-cold cell lysis buffer (Beyotime Institute of
Biotechnology, Haimen, China) for $30 \mathrm{~min}$. The lysates were centrifuged at $13,000 \mathrm{x}$ for $30 \mathrm{~min}$ at $4^{\circ} \mathrm{C}$. The supernatant was used for the subsequent assay. The enzyme activity was determined using a caspase-3 activity assay kit (Beyotime Institute of Biotechnology). The caspase-3 activity was determined by detecting the absorbance of p-nitroanilide at $405 \mathrm{~nm}$ with an Epoch microplate reader (BioTek Instruments, Inc.).

Detection of ROS levels. The peroxide-sensitive fluorescent probe, 2',7'-dichlorodihydrofluorescin diacetate (DCHF-DA; Beyotime Institute of Biotechnology), was used to measure intracellular ROS accumulation. The mesangial cells were initially incubated for $45 \mathrm{~min}$ at $37^{\circ} \mathrm{C}$ in PBS containing $10 \mu \mathrm{M}$ DCHF-DA to label intracellular ROS, prior to washing with PBS. Fluorescence was then observed when DCFH-DA was intracellularly oxidized to 2',7'-dichlorofluorescein (DCF). The fluorescence intensity of DCF was detected by flow cytometry using a BD FACSAria (BD Biosciences, Franklin Lakes, NJ, USA). The excitation and emission wavelengths were set at 488 and $525 \mathrm{~nm}$, respectively. BD CellQuest software (FACSDiva version 2.0; BD Biosciences) was used to assess flow cytometry data.

Terminal deoxynucleotidyl transferase dUTP nick-end labeling (TUNEL). TUNEL staining was performed using an in situ cell death detection kit (Roche Diagnostics GmbH, Mannheim, Germany), according to the manufacturer's protocol. Cells within a field were identified by 4',6-diamidino-2-phenylindole (Sigma-Aldrich) staining and the labeled glomerular mesangial cells were analyzed by confocal microscopy (FV300; Olympus Corporation, Tokyo, Japan). The percentage of apoptotic cells was determined by counting the number of apoptotic TUNEL-positive cells (out of a total of $>200$ cells in 5 random fields) and dividing by the total number of cells.

Annexin $V$ and propidium iodide (PI) staining assay. An annexin V/PI apoptosis detection kit (Beijing Biosea Biotechnology Co., Ltd., Beijing, China) was used to stain the cells with annexin V and PI, according to the manufacturer's protocol. The cells were subsequently centrifuged at $1,000 \mathrm{xg}$ for $5 \mathrm{~min}$ at $4^{\circ} \mathrm{C}$. The cell pellets were resuspended in binding buffer and treated with $5 \mu \mathrm{l}$ annexin $\mathrm{V}$ for $20 \mathrm{~min}$, then incubated with $10 \mu \mathrm{l}$ PI for $5 \mathrm{~min}$ in the dark. The rate of apoptosis was analyzed by flow cytometry (BD FACSAria; BD Biosciences) using the BD CellQuest software (FACSDiva v 2.0; BD Biosciences).

Western blot analysis. The cells were washed once with ice-cold PBS and lysed in buffer containing $1 \%$ protease inhibitor solution (Bio-Rad Laboratories, Inc., Hercules, CA, USA). The protein concentration was determined using a bicinchoninic acid protein assay reagent kit (Bio-Rad Laboratories, Inc.) and cellular proteins were extracted with lysis buffer containing $1 \%$ protease inhibitor solution. Next, protein lysates were separated on $15 \%$ sodium dodecyl sulfate-polyacrylamide gels and transferred onto polyvinylidene difluoride membranes (Thermo Fisher Scientific, Inc.). The membranes were blocked with 5\% non-fat dry milk (Bio-Rad Laboratories, Inc.) in PBS for $2 \mathrm{~h}$ at room temperature. The blots 
were incubated with the rabbit anti-LC3 primary antibody (dilution, 1:200) at $4^{\circ} \mathrm{C}$ overnight, followed by incubation with fluorescence-conjugated goat anti-rabbit immunoglobulin $\mathrm{G}$ (cat. no. A27033; Invitrogen; Thermo Fisher Scientific, Inc.) at a dilution of 1:10,000 for $1 \mathrm{~h}$ at room temperature. The protein bands were quantified using the Odyssey infrared imaging system (LI-COR, Inc., Lincoln, NE, USA).

Immunofluorescence. After $24 \mathrm{~h}$ of incubation with or without drugs, the culture media was removed and the cells were fixed in methanol for $5 \mathrm{~min}$ at room temperature. The cells were washed with PBS, permeabilized with $0.4 \%$ Triton X-100 in PBS for $1 \mathrm{~h}$, blocked with $5 \%$ bovine serum albumin (Gibco; Thermo Fisher Scientific, Inc.) for $1 \mathrm{~h}$ at room temperature, and incubated with rabbit anti-LC3 primary antibody (1:50) overnight at $4^{\circ} \mathrm{C}$. Subsequently, the cells were re-warmed and incubated in the dark with the goat anti-rabbit AlexaFluor594-conjugated secondary antibody (cat. no. R37117; Molecular Probes; Thermo Fisher Scientific, Inc.) at a dilution of 1:100 for $1 \mathrm{~h}$ at room temperature. The samples were washed with PBS and were subsequently mounted onto microscopic slides. The prepared slides were subsequently examined using a laser confocal microscope (Olympus FluoView 300; Olympus Corporation).

Examination of cell ultrastructure by transmission electron microscopy. The cells were incubated with or without drugs for $24 \mathrm{~h}$ and were subsequently fixed in $2.5 \%$ glutaraldehyde (Beyotime Institute of Biotechnology) overnight at $4^{\circ} \mathrm{C}$. The cells were post-fixed with $1 \%$ osmium tetroxide (Sigma-Aldrich) for $2 \mathrm{~h}$, washed in PBS and progressively dehydrated in a $10 \%$ graded series of 50 to $100 \%$ ethanol and propylene oxide. The blocks, which had been embedded in acrylic resin (Sigma-Aldrich) were cut into ultrathin sections $(50 \mathrm{~nm})$ with a microtome and were subsequently stained with saturated uranyl and lead citrate. The cell ultrastructure was examined using an H-7650 transmission electron microscope (Hitachi, Ltd., Tokyo, Japan).

Statistical analysis. The data are expressed as the mean \pm the standard error of the mean. Data analysis was performed using one-way analysis of variance followed by Bonferroni's test and Pearson correlation test. $\mathrm{P}<0.05$ was considered to indicate a statistically significant difference. GraphPad Prism software (version 6.0; GraphPad Software, Inc., La Jolla, CA, USA) was used to perform the data analysis.

\section{Results}

Activation of $\mathrm{HO}-1$ reverses $\mathrm{H}_{2} \mathrm{O}_{2}$-induced autophagy. Pathological induction of HO-1 was previously demonstrated to protect against renal injuries caused by ischemic acute renal failure, indicating that $\mathrm{HO}-1$ is involved in renal pathology (20). In the present study, an in vitro model of $\mathrm{H}_{2} \mathrm{O}_{2}$-induced glomerular mesangial cell injury was established to assess the protective effect of HO-1 activated by hemin, a HO-1 agonist. A concentration of $\sim 125 \mu \mathrm{M} \mathrm{H}_{2} \mathrm{O}_{2}$ was selected, as this concentration induces an appropriate level of cell damage (data not shown). Activation of HO-1 by the agonist hemin was demonstrated to reduce the expression of LC3 compared with
$\mathrm{H}_{2} \mathrm{O}_{2}$ treatment $(\mathrm{P}<0.001)$, which suggested that HO-1 effects $\mathrm{H}_{2} \mathrm{O}_{2}$-induced autophagy. Notably, the effect of hemin on autophagy when the intracellular ROS levels were overloaded was demonstrated to be dose-dependent (Fig. 1A). The electron microscopy and immunofluorescence results further verified this finding by demonstrating the effects of hemin on the size and quantity of autophagosomes (Fig. 1B and C). Inhibition of HO-1 by the antagonist, ZnPP, increased autophagy in the mesangial cells stimulated with $\mathrm{H}_{2} \mathrm{O}_{2}$. A previous study demonstrated that activation or inactivation of HO-1 does not alter basal autophagy (21). The results of the present study indicated that the effect of HO-1 on reducing autophagy may be ROS-dependent in glomerular mesangial cell.

HO-1-modulated autophagy is associated with oxidative stress relief and JNK activity. Pre-treatment with $125 \mu \mathrm{M} \mathrm{H}_{2} \mathrm{O}_{2}$ resulted in a significant increase in the levels of ROS in glomerular mesangial cells $(\mathrm{P}<0.001$; Fig. 2A). The addition of hemin reversed the increase in the ROS level to the basal level compared with $\mathrm{H}_{2} \mathrm{O}_{2}$ treatment $(\mathrm{P}<0.001)$. However, the addition of $\mathrm{ZnPP}$ resulted in no further increase in the ROS level compared with the levels induced by $\mathrm{H}_{2} \mathrm{O}_{2}$. These results suggested that effective free radical scavenging may require sufficient activation of HO-1. The oxidative stress relief following hemin treatment was concentration-dependant, as demonstrated by the detection of ROS intensity (Fig. 2B). The present study also demonstrated a significant proportional association between attenuated autophagy and oxidative stress relief. This association may be attributed to different degrees of HO-1 activation by hemin $(\mathrm{P}=0.0103$; Fig. $2 \mathrm{C})$. This result may indicate a dose-dependent intrinsic link between HO-1-mediated regulation of oxidative stress and autophagy.

The JNK agonist, anisomycin, significantly increased the LC3-II/LC3-I expression ratio in $\mathrm{H}_{2} \mathrm{O}_{2}$-treated glomerular mesangial cells (Fig. 2D). The stimulation of JNK activity by anisomycin eliminated the effect of hemin on autophagy relief. This finding suggested that the regulatory effect of hemin on LC3 expression is association with JNK signaling, which is a downstream signaling pathway induced by oxidative stress. This result demonstrated the importance of JNK signaling in the hemin-mediated association between attenuated autophagy and oxidative stress relief.

Attenuation of autophagy by hemin protects against $\mathrm{H}_{2} \mathrm{O}_{2}$-induced apoptosis. The specific autophagy agonist, rapamycin, was used in the present study to further investigate the specific function of activated HO-1 during autophagy in a cellular environment with an excessive release of free radicals. The activation of HO-1 by hemin protected against $\mathrm{H}_{2} \mathrm{O}_{2}$-induced cell apoptosis, as demonstrated by significantly increased cellular viability and downregulated activity of the apoptotic marker, caspase-3, compared with $\mathrm{H}_{2} \mathrm{O}_{2}$-treated glomerular mesangial cells $(\mathrm{P}<0.001$; Fig. 3A and $\mathrm{B})$. Additionally, TUNEL staining and flow cytometry assays supported these results (Fig. 3C and D). The present study demonstrated that co-treatment with the autophagy agonist rapamycin, which can induce excessive autophagy, partially counteracted the cytoprotective effect of HO-1. These results demonstrated that hemin attenuates excessive autophagy and 
A
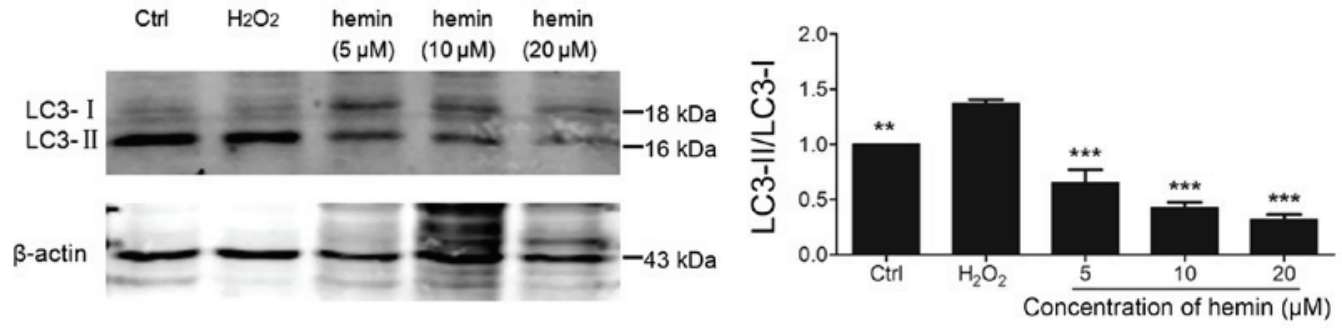

B
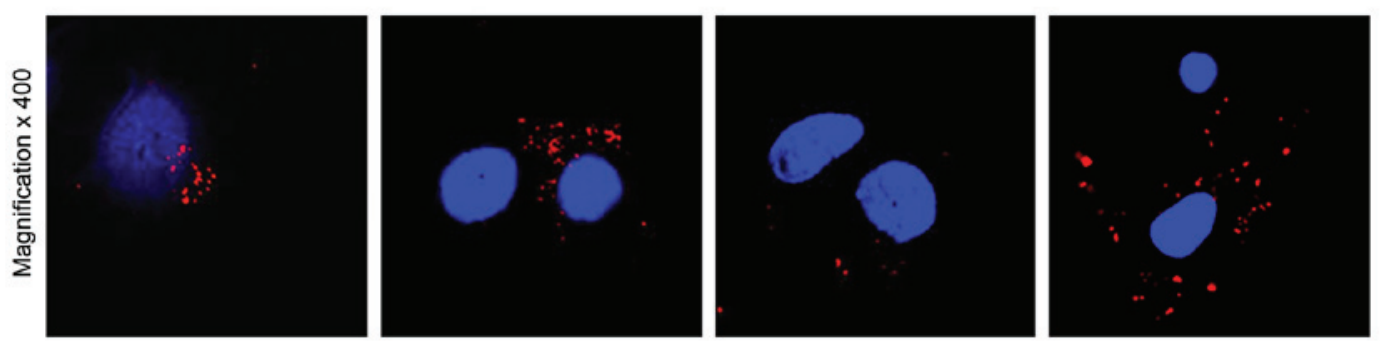

C

Ctrl

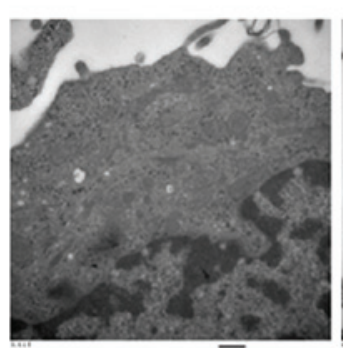

$\overline{500} \mathrm{~nm}$

Ctrl
$\mathrm{H}_{2} \mathrm{O}_{2}$

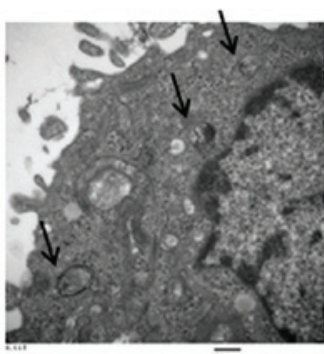

$\mathrm{H}_{2} \mathrm{O}_{2}$
$\mathrm{H}_{2} \mathrm{O}_{2}$ +hemin

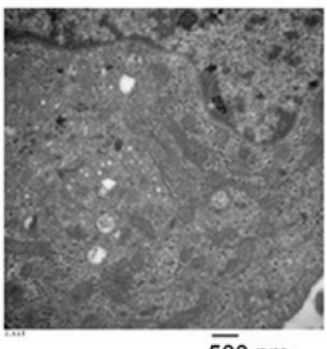

$\overline{500} \mathrm{~nm}$

$\mathrm{H}_{2} \mathrm{O}_{2}$ +hemin
$\mathrm{H}_{2} \mathrm{O}_{2}+\mathrm{ZnPP}$

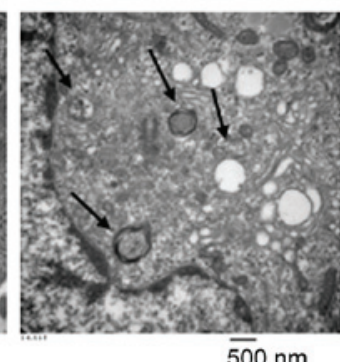

$\mathrm{H}_{2} \mathrm{O}_{2}+\mathrm{ZnPP}$

Figure 1. Effect of HO-1 zon autophagy-associated protein expression in $\mathrm{H}_{2} \mathrm{O}_{2}$-treated mesangial cells. (A) The effect of different concentrations of hemin on the LC3 II/LC3 I expression ratio $\left({ }^{* *} \mathrm{P}<0.01\right.$ and ${ }^{* * *} \mathrm{P}<0.001$ vs. $\left.\mathrm{H}_{2} \mathrm{O}_{2}\right)$. The data are presented as the mean \pm standard error of the mean. Results of (B) immunofluorescence and (C) electron microscopy experiments (the concentration of hemin was $10 \mu \mathrm{mol} / 1)$. Immunofluorescence analysis demonstrated that marked accumulation of LC3-positive red dots was observed in the $\mathrm{H}_{2} \mathrm{O}_{2}$ and $\mathrm{H}_{2} \mathrm{O}_{2}+\mathrm{ZnPP}$ treatment groups. Counterstaining was performed with 4',6-diamidino-2-phenylindole and the arrows indicate autophagosomes. LC3, microtubule associated protein 1 light chain 3; ZnPP, zinc protoporphyrin; HO, heme oxygenase.

provides cytoprotection against $\mathrm{H}_{2} \mathrm{O}_{2}$-induced cell injury via the inhibition of cell apoptosis.

\section{Discussion}

Glomerular mesangial cells are crucial for the regulation of glomerular filtration rate (22) and oxidative stress may impair this vital function of mesangial cells (23). By relieving oxidative stress, HO-1 is considered a potential therapeutic target in ischemic kidney disease $(2,24,25)$. Although the activation of HO-1 protects mesangial cells from oxidative stress-induced injury, the specific mechanism underlying the protective effect of $\mathrm{HO}-1$ remains elusive, particularly regarding cell autophagy (7). The present study identified the function of HO-1 during oxidative stress and autophagy in mesangial cells stimulated with $\mathrm{H}_{2} \mathrm{O}_{2}$.

It has previously been demonstrated that the overexpression of HO-1 reduces cisplatin-induced excessive autophagy in proximal tubule cells (3). In accordance with this previous finding, the current results further demonstrated that the pharmacological activation of HO-1 inhibits external oxidative stress-mediated autophagy in glomerular mesangial cells; however the inactivation of $\mathrm{HO}-1$ exacerbates the autophagic response. Taken together, these findings affirm the active modulation of HO-1 during autophagy. The HO-1-induced effect was also demonstrated to be dose-dependant, as HO-1 successfully inhibited the increasing autophagy caused by $\mathrm{H}_{2} \mathrm{O}_{2}$. However, no significant alteration in the basal autophagy level was detected in normal mesangial cells through the pharmacological intervention of HO-1 activity (21). This implies that the HO-1-mediated control of autophagy may be ROS-dependent. $\mathrm{HO}-1$ is the only endogenous source of carbon monoxide (CO), which is generated by $\mathrm{HO}-1$-catalyzed oxidative breakdown of heme (26). Generation of CO contributes to the regulation of cellular autophagy by HO-1. It was previously demonstrated that autophagy is activated by $\mathrm{CO}$ via $\mathrm{ROS}$ generation in the mitochondria, while Mito-TEMPO, a mitochondria-targeting antioxidant, reversed the effect of CO (27). Taken together, these results suggested that over-activation of $\mathrm{HO}-1$ failed to change the autophagy level under normal conditions in which only low abundance of heme was found, since the content of ROS was low.

HO-1 is one of the major endogenous antioxidants and can reduce the intracellular generation of ROS. As oxidative 
A

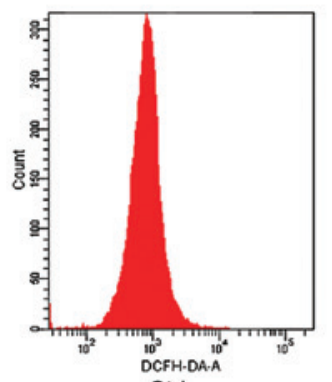

Ctrl

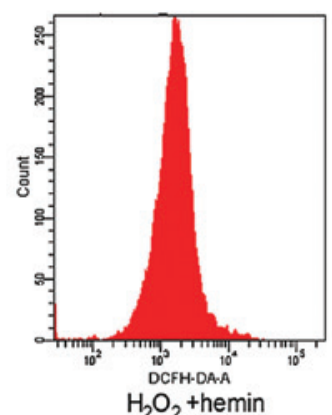

C

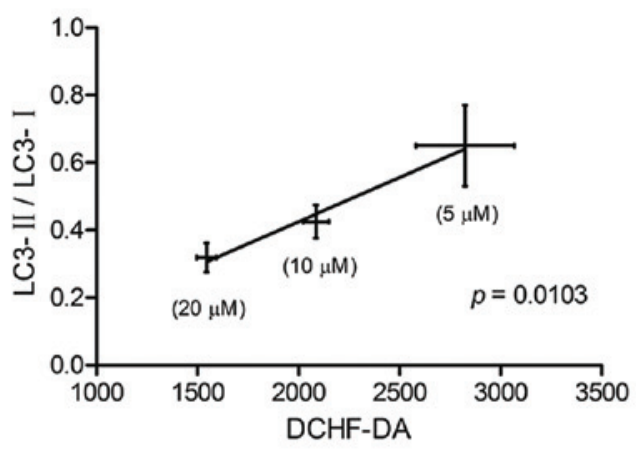

B
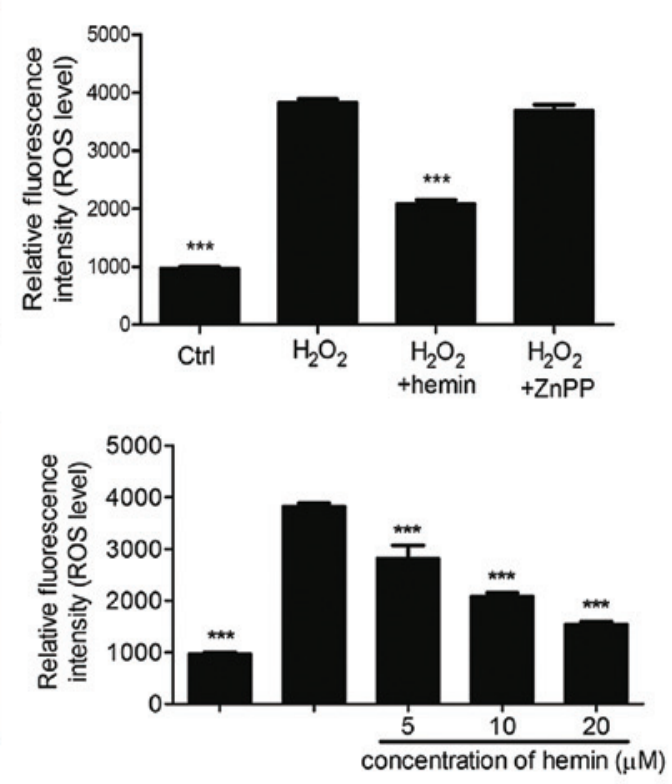

D
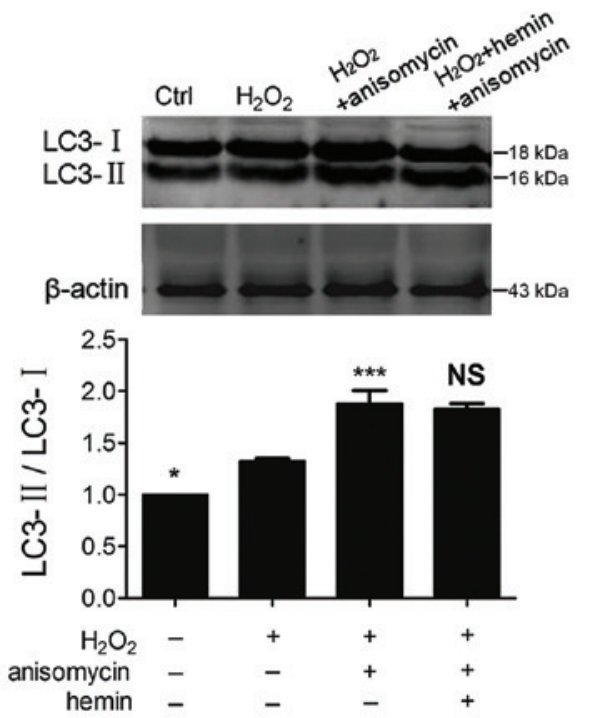

Figure 2. HO-1-modulated autophagy is proportional to ROS levels through a JNK-dependent mechanism. (A) Effect of hemin and ZnPP on the level of ROS in $\mathrm{H}_{2} \mathrm{O}_{2}$-treated mesangial cells ( ${ }^{* * * *} \mathrm{P}<0.001$ vs. $\mathrm{H}_{2} \mathrm{O}_{2}$ ). (B) The effect of different concentrations of hemin on the ROS level ( ${ }^{* * *} \mathrm{P}<0.001$ compared with $\mathrm{H}_{2} \mathrm{O}_{2}$ ). (C) Proportional association between the LC3 II/LC3 I expression ratio and the content of ROS following treatment with hemin at the concentration range of 5-20 $\mu \mathrm{M}$. (D) The JNK agonist anisomycin counteracts the effect of activated HO-1 on the LC3-II/LC3-I expression ratio in $\mathrm{H}_{2} \mathrm{O}_{2}$-treated mesangial cells $\left({ }^{*} \mathrm{P}<0.05,{ }^{* * *} \mathrm{P}<0.001\right.$ vs. $\left.\mathrm{H}_{2} \mathrm{O}_{2}\right)$. The data are presented as the mean \pm standard error of the mean. HO, heme oxygenase; JNK, c-Jun N-terminal kinase; ROS, reactive oxygen species; ZnPP, zinc protoporphyrin; LC3, microtubule associated protein 1 light chain 3; DCHF-DA, 2',7'-dichlorodihydrofluorescin diacetate; NS, not significant.

stress and autophagy are sequentially associated (28), the free radical scavenging role of $\mathrm{HO}-1$ activated by hemin may reverse the autophagy activating effect of $\mathrm{CO}$. Previous investigations $(3,29)$ and the present study confirmed this. The present study demonstrated that the inhibition of autophagy by hemin-activated HO-1 is significantly proportional to the effect of hemin on the scavenging of cellular free radicals. The current findings of HO-1-inhibited autophagy do not conflict with the results of previous studies performed in other types of cell, including hepatocytes and cardiomyocytes $(30,31)$. In these previous studies, the induction of HO-1 enhanced, rather than attenuated, autophagy. It was previously proposed that the different intracellular environments of cells from various sources may cause the opposing effects of HO-1 on autophagy. However, further experiments are required to perform this type of cell-based comparison under the same conditions and to clarify the potential mechanisms involved.

Increased levels of ROS alter autophagy in cells (32). The results of the present study demonstrated that HO- 1 is responsible for reducing the association between oxidative stress and autophagy. The HO-1 activation was demonstrated to reverse $\mathrm{H}_{2} \mathrm{O}_{2}$-induced cell injury significantly by exhibiting an effect in the levels of scavenging cellular free radicals and inhibiting autophagy in glomerular mesangial cells. Furthermore, the present study demonstrated that HO-1 may be regulated by JNK signaling. Previous studies 

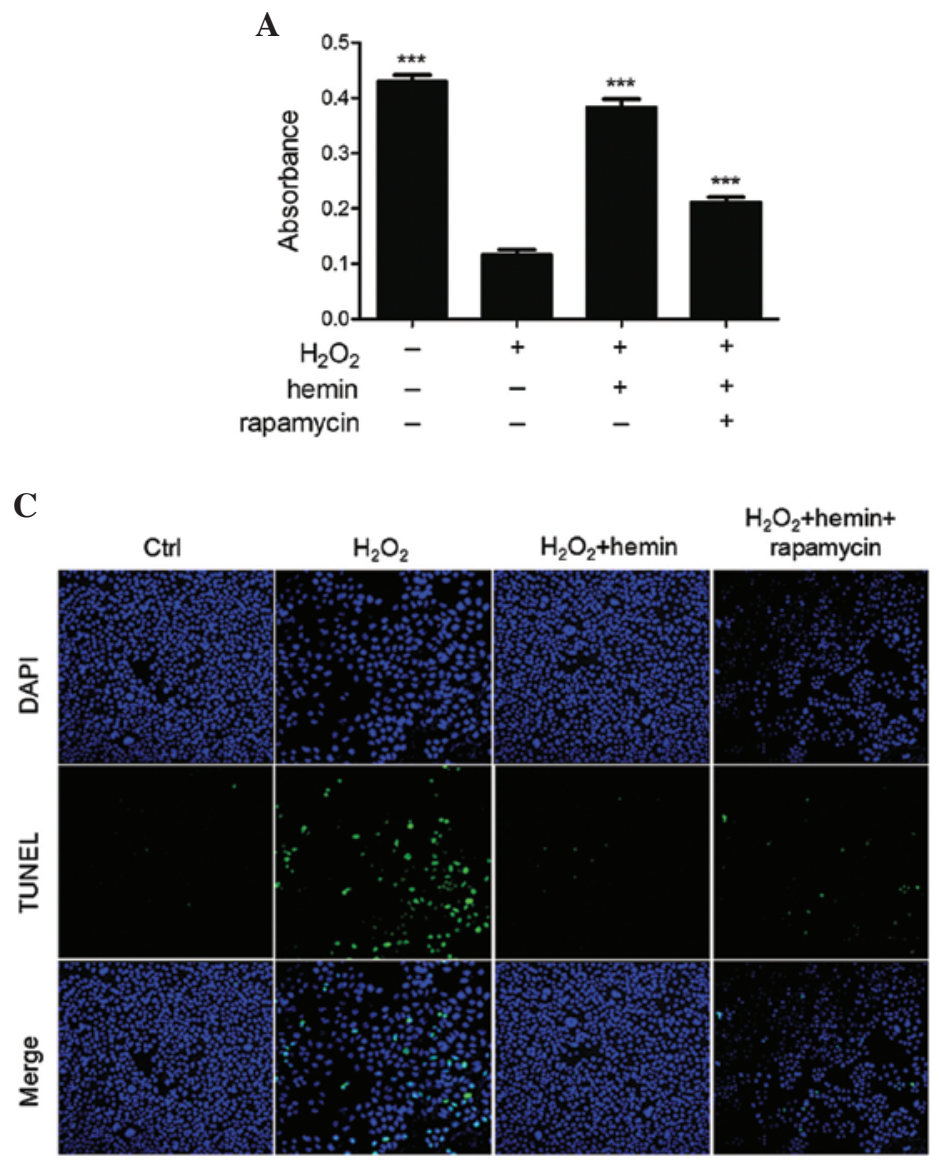

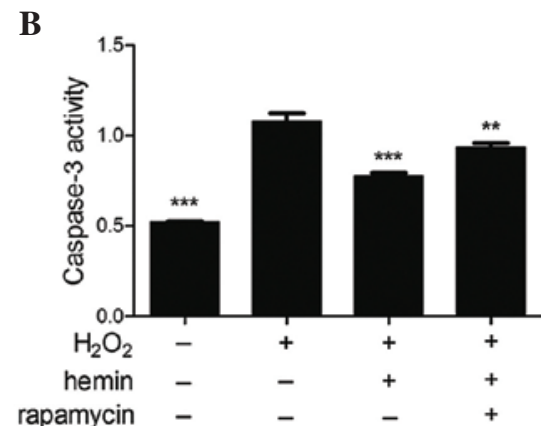

D
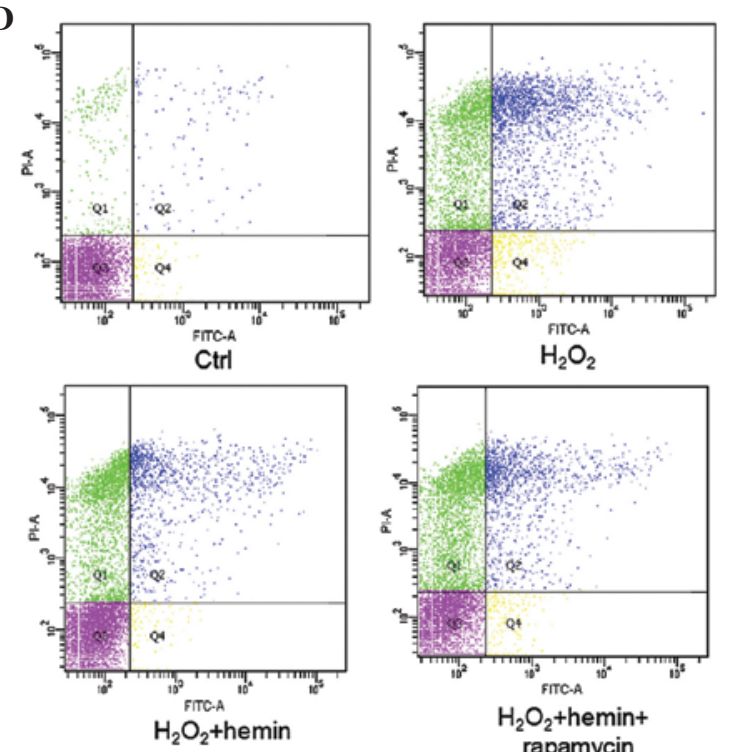

$\mathrm{H}_{2} \mathrm{O}_{2}$
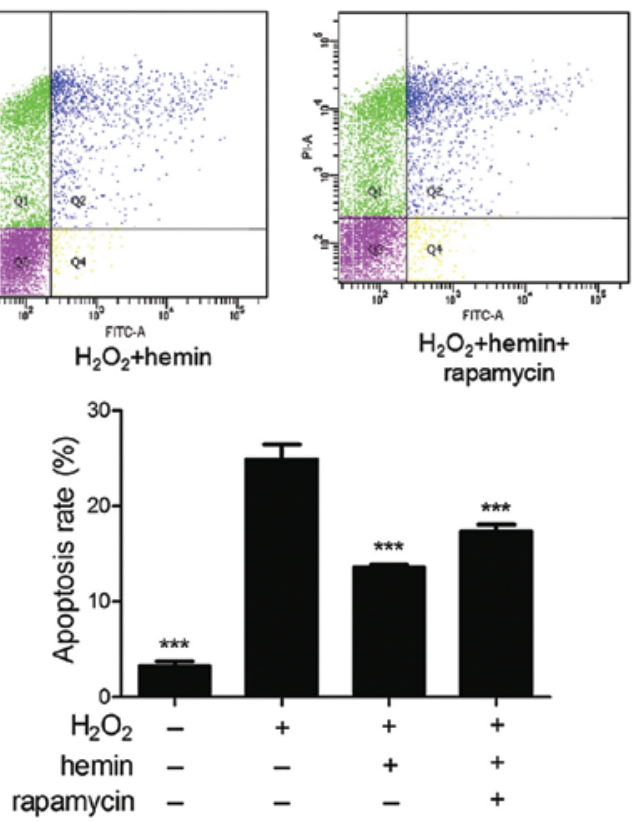

Figure 3. Effect of the HO-1 agonist hemin and the autophagy agonist rapamycin on apoptosis in $\mathrm{H}_{2} \mathrm{O}_{2}$-treated mesangial cells. The results of the (A) 3-(4,5-dimethyl-2-thiazolyl)-2,5-diphenyl-2-H-tetrazolium, (B) caspase-3, (C) TUNEL and (D) flow cytometry assays. TUNEL staining was performed to determine apoptosis (green dots represent apoptotic cells) and the results are expressed as apoptotic cell number (\%; $\mathrm{n}=5$ ). Flow cytometry was subsequently performed, for further validation, and data were expressed as apoptotic rate (\%) including the early (Q4) and late (Q2) apoptosis rate $(\mathrm{n}=3)$. Data are presented as the mean \pm standard error of the mean $\left({ }^{* *} \mathrm{P}<0.01,{ }^{* * *} \mathrm{P}<0.001\right.$ vs. $\left.\mathrm{H}_{2} \mathrm{O}_{2}\right)$. HO, heme oxygenase; TUNEL, terminal deoxynucleotidyl transferase dUTP nick-end labeling.

have demonstrated that the JNK pathway is one of the major ROS-modulated cell signals (33) and is involved in autophagy regulation (34). The present study demonstrated that, in addition to inducing the expression of HO-1 (17), JNK signaling may also have important functions in the HO-1-associated crosstalk between oxidative stress and autophagy. The results of the present study supported the findings reported by the previous study (35).

Apoptosis and autophagy are two complex, interlinked processes of cell death and degradation, respectively (36). The findings of the present study support the function of HO-1 as an important regulator during oxidative stress-induced autophagy and consequential apoptosis (3). Although self destruction may be used by cells as an important strategy during oxidative stress injury, natural selection applies specific methods that can be used by cells to survive in harsh environments. As an excessive increase in autophagy can harm cells (14), the successful relief of external ROS injury, the restoration of the autophagy level to the basal level, and the impediment of excessive autophagic flux demonstrate the important and unique functions of HO-1 during oxidative stress-induced autophagy. 
The present findings provided novel insights into the mechanisms underlying the HO-1-induced protection against oxidative stress-induced cell damage. The current results present additional information, which will improve the understanding of the specific functions of HO-1 during the cellular response to external stress.

\section{Acknowledgements}

The present study was supported by the Funds of Health and Family Planning Commission of Heilongjiang Province (grant nos. 2012-357, 2013-110 and 2014-379) and the Fund of Heilongjiang Postdoctoral Financial Assistance (grant no. LBH-Z14159).

\section{References}

1. Abraham NG, Cao J, Sacerdoti D, Li X and Drummond G: Heme oxygenase: The key to renal function regulation. Am J Physiol Renal Physiol 297: F1137-F1152, 2009.

2. Wagner M, Cadetg P, Ruf R, Mazzucchelli L, Ferrari P and Redaelli CA: Heme oxygenase-1 attenuates ischemia/reperfusion-induced apoptosis and improves survival in rat renal allografts. Kidney Int 63: 1564-1573, 2003

3. Bolisetty S, Traylor AM, Kim J, Joseph R, Ricart K, Landar A and Agarwal A: Heme oxygenase-1 inhibits renal tubular macroautophagy in acute kidney injury. J Am Soc Nephrol 21 1702-1712, 2010

4. Castilho A, Aveleira CA, Leal EC, Simões NF, Fernandes CR, Meirinhos RI, Baptista FI and Ambrósio AF: Heme oxygenase-1 protects retinal endothelial cells against high glucose- and oxidative/nitrosative stress-induced toxicity. PLoS One 7: e42428, 2012.

5. Zager RA, Johnson AC and Becker K: Plasma and urinary heme oxygenase-1 in AKI. J Am Soc Nephrol 23: 1048-1057, 2012.

6. Gozzelino R, Jeney V and Soares MP: Mechanisms of cell protection by heme oxygenase-1. Annu Rev Pharmacol Toxicol 50: 323-354, 2010.

7. Kapitsinou PP and Haase VH: HO-1 in control of a self-eating kidney. J Am Soc Nephrol 21: 1600-1602, 2010.

8. Klionsky DJ: Autophagy: From phenomenology to molecular understanding in less than a decade. Nat Rev Mol Cell Biol 8 : 931-937, 2007

9. Choi AM, Ryter SW and Levine B: Autophagy in human health and disease. N Engl J Med 368: 651-662, 2013.

10. Shintani T and Klionsky DJ: Autophagy in health and disease: A double-edged sword. Science 306: 990-995, 2004.

11. Mizushima N, Levine B, Cuervo AM and Klionsky DJ: Autophagy fights disease through cellular self-digestion. Nature 451: 1069-1075, 2008

12. Mizushima N and Komatsu M: Autophagy: Renovation of cells and tissues. Cell 147: 728-741, 2011.

13. Wang LT, Chen BL, Wu CT, Huang KH, Chiang CK and Hwa Liu S: Protective role of AMP-activated protein kinase-evoked autophagy on an in vitro model of ischemia/reperfusion-induced renal tubular cell injury. PLoS One 8: e79814, 2013.

14. Lim SW, Hyoung BJ, Piao SG, Doh KC, Chung BH and Yang CW: Chronic cyclosporine nephropathy is characterized by excessive autophagosome formation and decreased autophagic clearance. Transplantation 94: 218-225, 2012.

15. Jiang M, Liu K, Luo J and Dong Z: Autophagy is a renoprotective mechanism during in vitro hypoxia and in vivo ischemia-reperfusion injury. Am J Pathol 176: 1181-1192, 2010.

16. Wu H, Wang MC and Bohmann D: JNK protects Drosophila from oxidative stress by trancriptionally activating autophagy. Mech Dev 126: 624-637, 2009.
17. Haberzettl P and Hill BG: Oxidized lipids activate autophagy in a JNK-dependent manner by stimulating the endoplasmic reticulum stress response. Redox Biol 1: 56-64, 2013.

18. Rubio N, Verrax J, Dewaele M, Verfaillie T, Johansen T, Piette J and Agostinis P: p38(MAPK)-regulated induction of p62 and NBR1 after photodynamic therapy promotes autophagic clearance of ubiquitin aggregates and reduces reactive oxygen species levels by supporting Nrf2-antioxidant signaling. Free Radic Biol Med 67: 292-303, 2014.

19. Paccosi S, Musilli C, Mangano G, Guglielmotti A and Parenti A: The monocyte chemotactic protein synthesis inhibitor bindarit prevents mesangial cell proliferation and extracellular matrix remodeling. Pharmacol Res 66: 526-535, 2012.

20. Shimizu H, Takahashi T, Suzuki T, Yamasaki A, Fujiwara T, Odaka Y, Hirakawa M, Fujita H and Akagi R: Protective effect of heme oxygenase induction in ischemic acute renal failure. Crit Care Med 28: 809-817, 2000.

21. Xu J, Meng K, Zhang R, Yang H, Liao C, Zhu W and Jiao J: The use of functional chemical-protein associations to identify multi-pathway renoprotectants. PLoS One 9: e97906, 2014.

22. Stockand JD and Sansom SC: Glomerular mesangial cells: Electrophysiology and regulation of contraction. Physiol Rev 78: 723-744, 1998.

23. Jaimes EA, Hua P, Tian RX and Raij L: Human glomerular endothelium: Interplay among glucose, free fatty acids, angiotensin II, and oxidative stress. Am J Physiol Renal Physiol 298: F125-F132, 2010.

24. Takahashi T, Morita K, Akagi R and Sassa S: Protective role of heme oxygenase-1 in renal ischemia. Antioxid Redox Signal 6: 867-877, 2004.

25. Shi Q, Feng YN, Fang J and Xu K: Pretreatment with glutamine attenuates anoxia/reoxygenation injury of human proximal renal tubular epithelial cells via induction of heme oxygenase-1. Pharmacology 84: 1-8, 2009.

26. Ryter SW and Choi AM: Regulation of autophagy in oxygen-dependent cellular stress. Curr Pharm Des 19: 2747-2756, 2013.

27. Salahudeen AK, Yang M,Huang H,Dore S and Stec DE: Fenoldopam preconditioning: Role of heme oxygenase-1 in protecting human tubular cells and rodent kidneys against cold-hypoxic injury. Transplantation 91: 176-182, 2011.

28. Kiffin R, Bandyopadhyay U and Cuervo AM: Oxidative stress and autophagy. Antioxid Redox Signal 8: 152-162, 2006.

29. Li CW, Lin YF, Liu TT and Wang JY: Heme oxygenase-1 aggravates heat stress-induced neuronal injury and decreases autophagy in cerebellar Purkinje cells of rats. Exp Biol Med (Maywood) 238: 744-754, 2013.

30. Yun N, Cho HI and Lee SM: Impaired autophagy contributes to hepatocellular damage during ischemia/reperfusion: Heme oxygenase-1 as a possible regulator. Free Radic Biol Med 68: 168-177, 2014.

31. Zhao Y, Zhang L, Qiao Y, Zhou X, Wu G, Wang L, Peng Y, Dong X, Huang $\mathrm{H}$, Si L, et al: Heme oxygenase-1 prevents cardiac dysfunction in streptozotocin-diabetic mice by reducing inflammation, oxidative stress, apoptosis and enhancing autophagy. PLoS One 8: e75927, 2013.

32. Scherz-Shouval R, Shvets E, Fass E, Shorer H, Gil L and Elazar Z: Reactive oxygen species are essential for autophagy and specifically regulate the activity of Atg4. EMBO J 26: 1749-1760, 2007.

33. Leonarduzzi G, Sottero B, Testa G, Biasi F and Poli G: New insights into redox-modulated cell signaling. Curr Pharm Des 17: 3994-4006, 2011.

34. Lorin S, Pierron G, Ryan KM, Codogno P and Djavaheri-Mergny M: Evidence for the interplay between JNK and p53-DRAM signalling pathways in the regulation of autophagy. Autophagy 6: 153-154, 2010.

35. Kietzmann T, Samoylenko A and Immenschuh S: Transcriptional regulation of heme oxygenase-1 gene expression by MAP kinases of the JNK and p38 pathways in primary cultures of rat hepatocytes. J Biol Chem 278: 17927-17936, 2003.

36. Thorburn A: Apoptosis and autophagy: Regulatory connections between two supposedly different processes. Apoptosis 13: 1-9, 2008. 